\section{alloSCT bei AML oder MDS: dosisreduzierte Konditionierung erhöht die Rezidivrate}

Die optimale Intensität der Konditionierung vor allogener Stammzelltransplantation (alloSCT) ist nicht bekannt. Erste Ergebnisse mit dosisreduzierten Konditionierungsregimes (RIC) ließen vermuten, dass diese gegenüber myeloablativen Regimes (MAC) die therapiebedingte Mortalität (TRM) reduzieren und damit das Gesamtüberleben (OS) verlängern könnten.

$\mathrm{D}$ iese Hypothese wurde in einer Phase-III-Studie bei 272 Patienten mit akuter myeloischer Leukämie (AML) oder myelodysplastischem Syndrom (MDS) geprüft, die randomisiert MAC $(\mathrm{n}=135)$ oder RIC $(\mathrm{n}=137)$ erhielten, gefolgt von einer alloSCT von HLA-gematchten verwandten oder nichtverwandten Spendern. Primärer Endpunkt war das OS nach 18 Monaten, sekundäre Endpunkte rezidivfreies Überleben (RFS) und TRM.
Ursprünglich sollten 356 Patienten an der Studie teilnehmen. Die Aufnahme wurde jedoch nach 271 Patienten durch das unabhängige Daten- und Sicherheitsboard gestoppt, da sich unter RIC eine unerwartet hohe Rezidivneigung gezeigt hatte ( 48,3 vs. $13,5 \%$ unter MAC; $\mathrm{p}<0,001)$.

Nach 18 Monaten betrug die OS-Rate der Patienten im RIC-Arm 67,7 versus $77,5 \%$ im MAC-Arm. Die Differenz von 9,8\% zugunsten des MAC-Arms war je-

doch für die Gesamtpopulation nicht statistisch signifikant $(\mathrm{p}=0,07)$. Unter der RIC war die TRM deutlich niedriger $(4,4$ vs. $15,8 \% ; p=0,002)$. Infolge der hohen Rezidivrate nach der RIC belief sich die Rate für das RFS unter RIC aber nur auf $47,3 \%$ im Vergleich zu $67,8 \%$ unter $\operatorname{MAC}(\mathrm{p}<0,01)$.

Fazit: Das OS war unter MAC höher als unter RIC; diese Differenz war nicht signifikant. Die RIC führte zu einer niedrigeren TRM, aber höheren Rezidivraten. Bezüglich des RFS war die MAC signifikant im Vorteil. Die Daten sprechen dafür, dass die myeloablative Konditionierung vor der alloSCT bei Patienten mit AML oder MDS der Standard bleiben muss.

Brigitte Schalhorn

Scott BL et al. Myeloablative versus reduced-intensity hematopoietic cell transplantation for acute myeloid leukemia and myelodysplastic syndromes. J Clin Oncol. 2017;35(11):1154-61.

ten waren median 2 -fach vorbehandelt, praktisch alle hatten bereits einen CD20Antikörper und $\geq 90 \%$ eine FludarabinKombination erhalten.

Nach median 14 Monaten betrug das mediane PFS unter IDE 20,8, unter Placebo 11,3 Monate (Hazard Ratio [HR] $0,33 ; p=0,0001)$. Praktisch alle Subgruppen erreichten unter IDE ein längeres PFS, darunter auch Patienten mit del(17p) oder TP53-Mutation (11,3 vs. 8,3 Monate; HR 0,47; $<$ 0,0001; Abb. 1). Auch das OS war unter der Addition von IDE zu BR signifikant länger (Median nicht erreicht versus 30,6 Monate; HR 0,62; $\mathrm{p}=0,031$ ).

Fazit: Die IDE-Kombination senkt im Vergleich zur BR-Standardtherapie das Risiko für Progression und Tod klinisch relevant, auch bei Patienten mit refraktärer oder rezidivierter CLL mit oder ohne Risikofaktoren. Andererseits müssen häufiger auftretende gravierende Nebenwirkungen, v. a. Infektionen, beobachtet und therapiert werden. Brigitte Schalhorn

Zelenetz AD et al. Idelalisib or placebo in combination with bendamustine and rituximab in patients with relapsed or refractory chronic lymphocytic leukaemia: interim results from a phase 3 , randomised, double-blind, placebo-controlled trial. Lancet Oncol. 2017(18):297-311.

Abb. 1: Auch CLL-Patienten mit del(17p) oder TP53-Mutation lebten unter Idelalisib länger progressionsfrei.
(IGHV, del[17p], TP53-Mutation) sowie „refraktär“ versus „rezidiviert“ stratifigressionsfreie Überleben (PFS), sekundäre Endpunkte Remissionsraten und Gesamtüberleben (OS). Die 416 Patien- 